\title{
Ein Stethoskop zum Überprüfen von Brücken
}

Beim Überprüfen der Tragsicherheit einer bestehenden Brücke können dank Messungen die zur Berechnung der Beanspruchung entwickelten Modelle kalibriert werden. Im vorliegenden Aufsatz wird ein neues System von Dehnmessstreifen vorgestellt, welches den erforderlichen Zeitaufwand für die Messungen erheblich reduziert. In wenigen Minuten sind das System installiert und die Messungen durchgeführt. Dies bedeutet für den Ingenieur ein neues Werkzeug, das so nützlich ist, wie für den Arzt das Stethoskop. Einige Anwendungsmöglichkeiten und Vorteile, welche das neue Messsystem aufweist, werden an einem Pilotprojekt, der Kirchenfeldbrücke in Bern, die 1883 in Betrieb genommen wurde, aufgezeigt.

Wie die Kirchenfeldbrücke in Bern (Bild 1), müssen heutzutage viele Kunstbauten auf ihre Tragsicherheit und hinsichtlich Ermüdung überprüft werden, um die Nutzungsdauer zu verlängern oder die zulässigen Strassenlasten zu erhöhen. Im Gegensatz zu neuen Bauwerken ist es bei bestehenden möglich, die Ergebnisse aus den Berechnungsmodellen mit gemessenen Werten an der Brücke zu vergleichen. Diese Vergleiche ermöglichen eine Verfeinerung der Modelle und können oft verhindern, dass ein aufwendiger und teurer Eingriff am Bauwerk erfolgen muss. Das Anwenden herkömmlicher Dehnmessstreifen ist aber zeitaufwendig und somit auch teuer, was die Bauherren häufig davon abhält, solche Messungen anzuordnen.

Ein neues, in Japan entwickeltes System von Dehnmessstreifen, welches über Reibung funktioniert, ermöglicht eine enorme Verminderung des Messaufwandes. Deshalb können mit diesem neuen System grosse finanzielle Einsparungen bei den Messungen gemacht werden, was dessen Anwenden beim Überprüfen der Tragsicherheit bestehender Bauten bedeutend interessanter macht.

\section{Das neue Messsystem}

Bild 2 zeigt das neue Messsystem, welches von der Tokioter Gesellschaft Tokyo Sokki Kenkyijo zusammen mit der Nagoya Universität in Japan entwickelt wurde [1], [2]. In einem kleinen Stahlzylinder von ungefähr $6 \mathrm{~cm}$ Länge befindet sich ein Stempel. Daran ist ein Dehnmessstreifen angebracht. Da der Zylinder am unteren Ende magnetisch ist, kann er problemlos an einem Stahlelement befestigt werden. Der Stempel wird dabei durch eine Feder an die Stahlkonstruktion gepresst und misst die Dehnung über die dadurch entstandene Haftreibung. Ein wesentlicher Vorteil dieses Systems ist, dass die aufwendige Arbeit des Aufklebens auf den Stahl, wie es bei den herkömmlichen Messstreifen der Fall ist, entfällt. Es genügt, den Magnet an der gewünschten Messstelle zu fixieren, und die Messung kann beginnen.

Die Versuchsmessungen, welche das Institut für Stahlbau (ICOM) der ETH Lausanne durchgeführt hat [3], bestätigen die Angaben des Herstellers [1], dass nämlich die gemessenen Verformungen unabhängig davon sind, ob das Stahlelement angestrichen ist oder nicht. Mit diesen Ergebnissen entfällt das Abschleifen der Farbe und somit muss nach Beenden der Messungen auch nicht mehr nachgebessert werden. Weitere Versuche am ICOM [4] haben gezeigt, dass diese Messstreifen die Möglichkeit haben, mit guter Genauigkeit die Schwingungen eines Trägers (Bild 3) zu messen und daraus dessen Eigenfrequenz und Dämpfungsrate abzuleiten.

Um Verformungen auf einem Betonelement zu messen, muss lediglich ein dünnes Stahlblech an die Betonoberfläche geklebt werden; anschliessend kann der Dehnmessstreifen auf dem Blech angebracht werden. Die Befestigung des Bleches erfolgt zum Beispiel mit Hilfe eines Schnellklebers. Die Ergebnisse dieser Messmethode wurden mit den Resultaten aus Omega-Messstreifen, welche normalerweise für Verformungsmessungen an Betonelementen eingesetzt werden, verglichen. Die Resultate zeigen, dass kein signifikanter 
Unterschied zwischen den beiden Messmethoden besteht.

Die Reibungsmessstreifen liefern also sowohl für gestrichene oder ungestrichene Stahloberflächen als auch für Betonoberflächen zuverlässige Resultate. Ausserdem können mit den Messstreifen statische und dynamische Messungen durchgeführt werden. Dank dieser Eigenschaften, zusammen mit der Möglichkeit des problemlosen Versetzens, konnte an der Kirchenfeldbrücke in Bern ein erfolgreiches Pilotprojekt durchgeführt werden. Es folgten weitere Einsätze vor Ort an der Aarebrücke zwischen Felsenau und Koblenz (Kt. Aargau) und an der Kornhausbrücke in Bern.

\section{Pilotprojekt Kirchenfeldbrücke}

Bild 4 zeigt Ansicht und Querschnitt der Kirchenfeldbrücke in Bern. Es handelt sich um eine genietete Brücke. Trotz verschiedener Inspektionen und Eingriffen stellt sich immer wieder die Frage nach Tragsicherheit und Ermüdungswiderstand in Bezug auf die erhöhten Verkehrslasten (Tram) im Verlaufe der Jahre. In der Tat wurde die Nutzungsdauer immer wieder verlängert.

Das Büro Hager + Bettschen in Bern wurde von den Verkehrsbetrieben Bern, BERNMOBIL, beauftragt, den Grenzzustand der Tragsicherheit des Bauwerks zu überprüfen. Ausserdem hat BERNMOBIL bei diesem Auftrag das ICOM sowohl als Experte zugezogen als auch mit der Überprüfung der Berechnungsmodelle anhand einer Messreihe an kritischen Tragelementen beauftragt [3].

Im Verlaufe eines Tages wurden an 10 Messstellen, davon 7 auf der Stahlstruktur des Brückenträgers und 3 auf der Unterseite der Betonplatte, jeweils zwei Reibungsmessstreifen angebracht (Bilder 5 und 6). Die Messstreifen wurden ohne Gerüst von Bergführern befestigt. Pro Messstelle erfolgten 3 bis 5 Messungen. Die in Bild 7 dargestellten Dehnungen wurden von zwei Messstreifen im selben Abschnitt gemessen. Die Überfahrt von zwei Fahrzeugen, einem Bus auf der einen und einem Tram auf der anderen Fahrspur, ist klar erkennbar. Der Vergleich zwischen den Messungen und den Resultaten aus der Berechnung hat gezeigt, dass die wirklichen Dehnungen kleiner sind als die berechneten. Um diese Beobachtung in der Berechnung zu berücksichtigen, kann ein Modellfaktor zur Reduktion der berechneten Beanspruchung eingeführt werden. Für die Stahlkonstruktion der Kirchenfeldbrücke ist dies ein
Modellfaktor von 0,80 . Ausserdem konnte mit den Messungen gezeigt werden, dass die vorhandene Biegung in den Diagonalen der Querträger (Winkelbleche) aufgrund der Exzentrizität der Stabachse zur Fachwerksebene kleiner ist als die theoretische Biegung (berechnet mit der Stabnormalkraft mal deren Exzentrizität). Für dieses Verhalten wurde in den Berechnungen noch ein weiterer Reduktionsfaktor eingeführt; im Beispiel der Kirchenfeldbrücke beträgt er rund 0,75.

Aufgrund der Messungen konnten die berechneten Beanspruchungen in der Stahlstruktur auf $80 \%$ reduziert werden, und zwar sowohl für den Grenzzustand der Tragsicherheit als auch für die Ermüdung. Die Biegemomente in den Diagonalen konnten sogar auf $60 \% \quad(=0,80 \cdot 0,75)$ des berechneten Wertes minimiert werden. Dank dieser Erkenntnisse konnte ein grosser Eingriff - das Ersetzen mehrerer Diagonalen des Brückenträgers sowie die Verstärkung mehrerer Gurte des Fachwerkquerträgers - vermieden werden.

Da der Zugang zu den Messstellen relativ schwierig ist, benötigte es zum Durchführen dieser Messungen etwa drei bis fünf Personen auf dem Bauwerk. Jede Messstelle beanspruchte 30 bis 40 Minuten. Darin sind der Zugang zur Messstelle, das Anbringen und Verkabeln der zwei Messstreifen, die Messung selbst (für jede Messstelle 3 bis 5 Messungen von je einer Minute) und die Demontage der Messstreifen enthalten. Es ist möglich, die Verformungen an einem Dutzend Messstellen innerhalb eines Arbeitstages zu messen. Je nach Wunsch des Auftraggebers können die Daten ausgewertet oder in unverarbeitetem Zustand übergeben werden. Beim herkömmlichen System der Dehnmessstreifen wären nur schon zum Aufbau des Gerüsts, zum Abschleifen der Farbe und zum Ankleben der Messstreifen 100 Arbeitsstunden nötig gewesen. Ausserdem kämen noch 100 Stunden für die Messungen, das Nachbessern der Farbe, das Entfernen des Gerüsts und die Datenauswertung dazu. Das wirtschaftliche Interesse an den Reibungsmessstreifen ist also offensichtlich, denn es können sowohl grössere Eingriffe an der Struktur vermieden als auch wesentliche Messkosten eingespart werden.

\section{Weitere Anwendungen}

In Bern wurde mit der Kornhausbrücke eine weitere über 100-jährige Brücke auf ihren Grenzzustand der 
Tragfähigkeit getestet. Anhand der Messungen mit den Reibungsmessstreifen konnte wie schon bei der Kirchenfeldbrücke ein Modellfaktor und die effektive Exzentrizität der gemessenen Elemente definiert werden.

An der Aarebrücke zwischen Leuggern / Felsenau und Koblenz (Kt. Aargau) wurden ebenfalls Messungen durchgeführt [5]. Vor kurzem wurden an der Brücke Renovationsarbeiten durchgeführt. Die Fahrbahn war sehr weich was grosse Spannungsdifferenzen in den Bogendiagonalen zur Folge hatte. Um die globale Steifigkeit des Systems zu erhöhen wurde beidseits der Fahrbahn je ein Versteifungsfachwerk eingebaut. Ausserdem wurde die schmale Fahrbahn mit einer breiteren, nun symmetrischen, orthotropen Platte ersetzt. Mit den Messungen wird das reale Tragverhalten sowohl mit den Berechnungen des Ingenieurs als auch mit den Messungen an der früheren Brücke verglichen. Dafür wurden an einigen ausgewählten Elementen wie Bogendiagonalen (Bild 8), Hänger, Querträger und orthotrope Platte (Bild 9) Reibungsmessstreifen angebracht. Der dynamische Beiwert kann dank der Messungen ebenfalls bestimmt werden. Die Messdaten können auch in Zukunft zum Bestimmen der Schadensakkumulation und der Restnutzungsdauer benutzt werden. Es ist somit möglich, dank diesem einfachen Hilfsmittel mit geringem Aufwand wichtige Erkenntnisse über das Bauwerk zu gewinnen.

\section{Anwendungsbereich}

Die möglichen Anwendungsbereiche der Reibungsmessstreifen zur Überprüfung bestehender Tragwerke sind zahlreich. Die wesentlichen sind :

- Eichen und Kalibrieren von Berechnungsmodellen für den Nachweis der Tragsicherheit, der Gebrauchstauglichkeit und des Ermüdungswiderstandes (Fall der Kirchenfeldbrücke).

- Bestimmen der wirklichen Amplitude der Spannungswechsel und Bestimmen der Schadensakkumulation, mit der anschliessend die Restnutzungsdauer der Stahlstruktur auf Ermüdung ermittelt werden kann (Möglichkeit für Aarebrücke).

- Messen der Eigenfrequenz und der Dämpfungsrate eines Bauwerkes (zum Beispiel einer schwingungsanfälligen Fussgängerbrücke).

\section{Schlussfolgerungen}

Mit Hilfe der Reibungsmessstreifen können Verformungen an Stahl- (gestrichen oder ungestrichen) und an Betonoberflächen schnell und zuverlässig gemessen werden. Die Zuverlässigkeit und die Genauigkeit wurden vom Institut für Stahlbau ICOM getestet. Die Einfachheit der Anwendung wurde am Beispiel der Kirchenfeld und der Felsenaubrücke veranschaulicht.

Mit den Messungen können die Berechnungsmodelle kalibriert und Informationen über das Verhalten und die Eigenschaften des Tragwerks bereitgestellt werden und somit genauere Angaben über das Verhalten und den Zustand des Tragwerks gemacht werden. Dies kann oft dazu führen, dass Verstärkungen oder Erneuerungen am Tragwerk vermieden werden können. Dieses «Stethoskop » ermöglicht es den Ingenieuren, in gewissen Fällen, für die bestehende Brücke eine längere Nutzungsdauer anzuordnen, ohne dass die Kosten dieser "Geriatrie » in die Höhe schiessen.

Dank

Die Autoren möchten sich bei Herrn Prof. Kentaro Yamada von der Nagoya Universität für die Präsentation und die Einführung des neuen Messsystems am ICOM bedanken. Ebenfalls möchten wir uns bei BERNMOBIL und dem Büro Hager + Bettschen sowie dem Departement für Bau, Verkehr und Umwelt des Kantons Aargau für ihr Vertrauen, welches sie uns im Rahmen dieser Messungen an der Kirchenfeld- und an der Felsenaubrücke entgegengebracht haben, bedanken. Weiterer Dank geht an Yves Rey, Sylvain Demierre, Hans-Jakob Reist, Gilles Guignet und Delphine Chou für die Vorbereitungen und die Ausführung der Versuche im Labor und vor Ort.

\section{Literatur}

[1] Tokyo Sokki Kenkyujo Co. Ltd., Strain Checker FGMH-1, Product documentation, 2005.

[2] Ojio, T., Yamada, K., Saito, Y., Shiina, S., Strain Checker: stethoscope for bridge engineers, in: Proceedings of IABMAS 06, Third International Conference on Bridge Maintenance, Safety and Management, Porto, Juli 2006. 
[3] Rey, Y., Thomann, M., Nussbaumer, A., Mesures sur le pont de Kirchenfeld, Rapport de mandat IC 934-1, Laboratoire de la construction métallique, Ecole polytechnique fédérale de Lausanne, 2006.

[4] Chou, D., Poutre Rolex, Jauges FGMH-1, Système porteur mixte avec sous-tirant, Projet de semestre, Laboratoire de la construction métallique, Ecole polytechnique fédérale de Lausanne, 2006.

[5] Sommerstein, T., Hirt, M.A., Messungen an der Aarebrücke Leuggern / Felsenau - Koblenz, Bericht Nr. IC 667-8, Laboratoire de la construction métallique, Ecole polytechnique fédérale de Lausanne, 2007. 

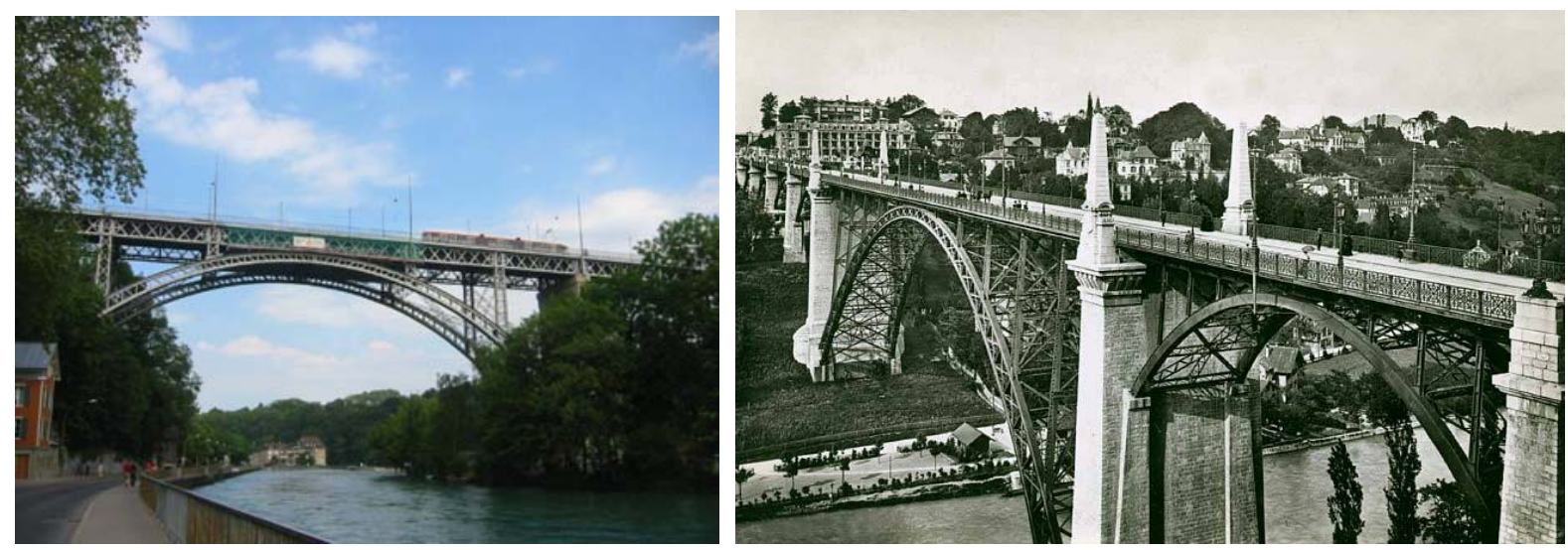

Bild 1 : Brücken von Bern - Kirchenfeldbrücke (Foto von 2004) und Kornhausbrücke (Foto von 1914)
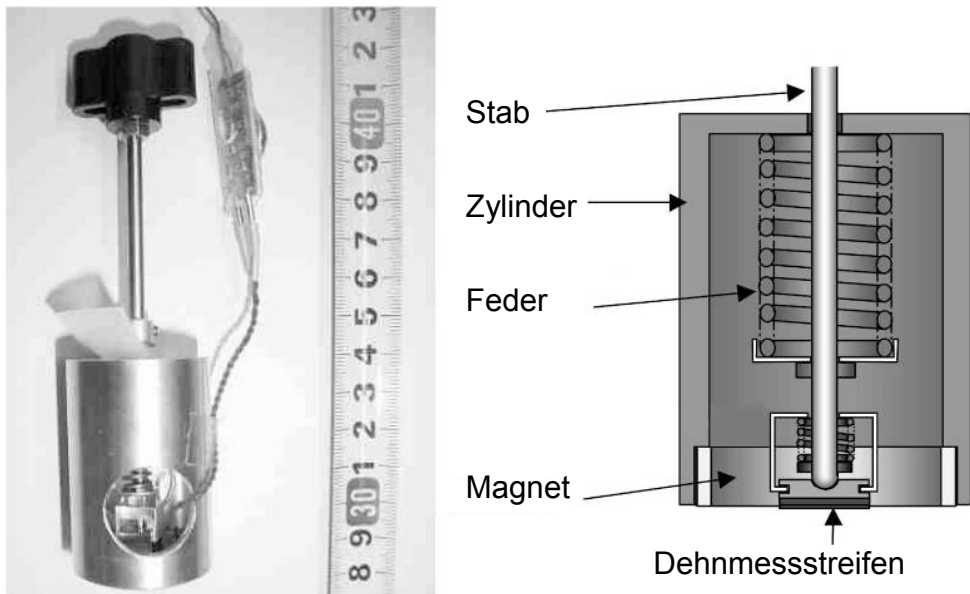

Bild 2 : Aufbau eines Reibungsmessstreifens [2]

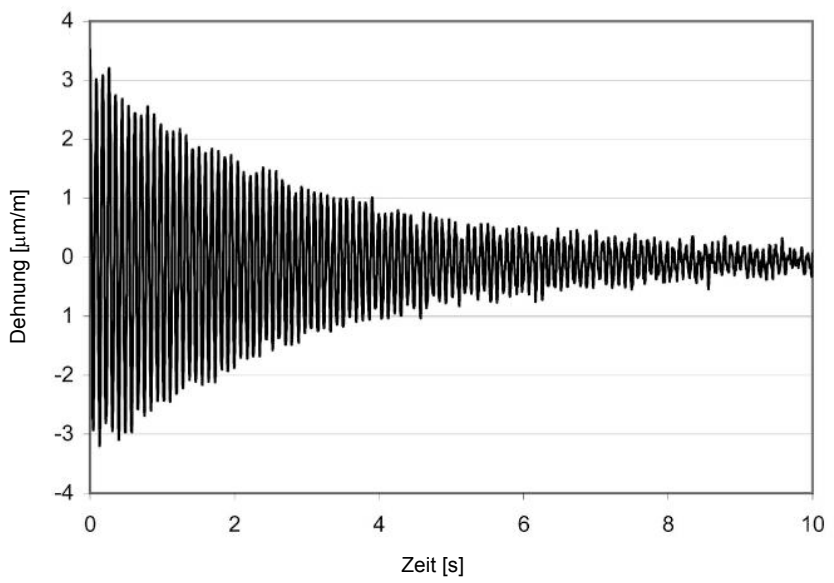

Bild 3 : Messung der Vibrationen an einem Verbundträger (Übertragungsfrequenz : 200 Hz), [4] 


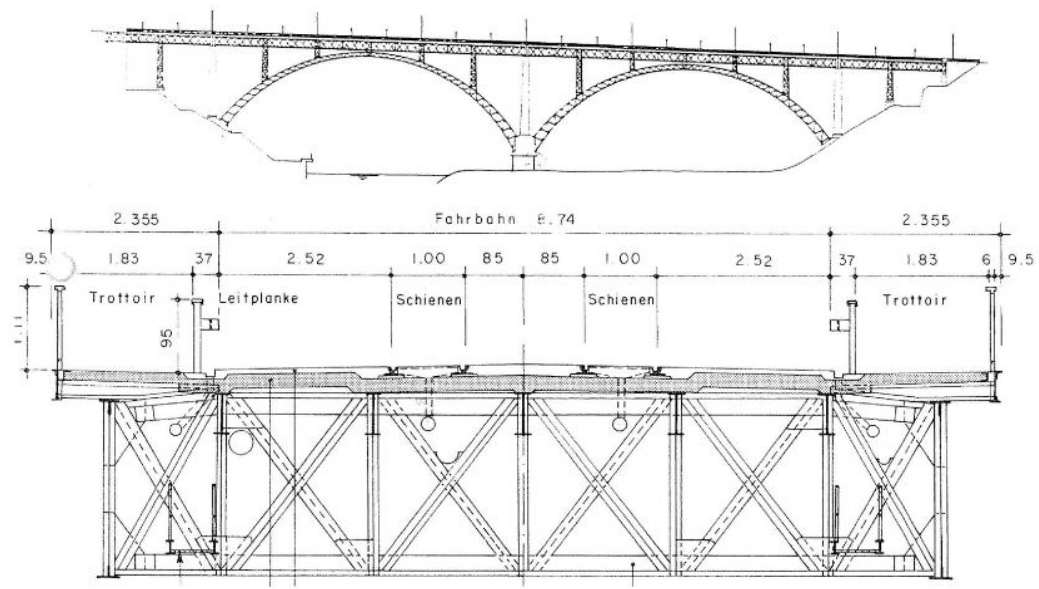

Bild 4 : Kirchenfeldbrücke in Bern : Längenprofil und Querträger

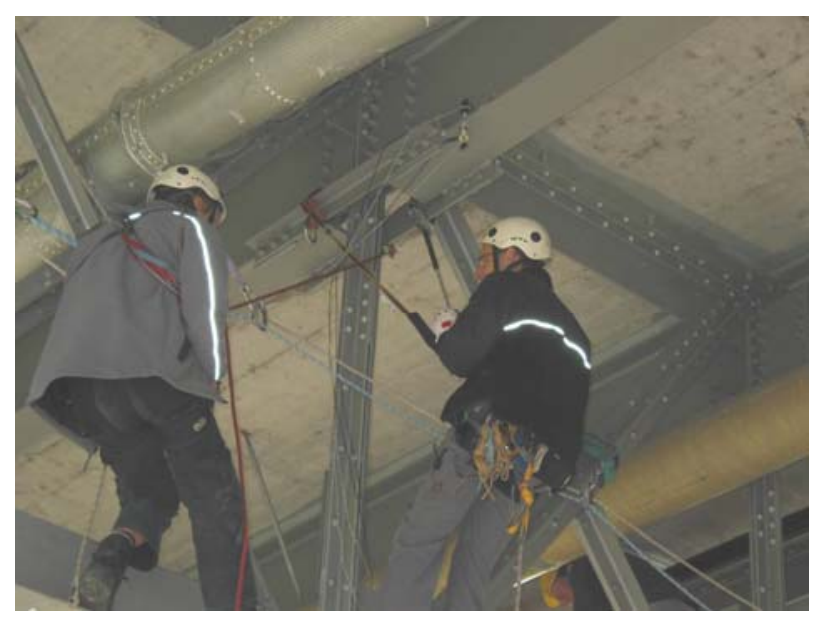

Bild 5 : Bergführer während Anbringen der Reibungsmessstreifens an Querträger

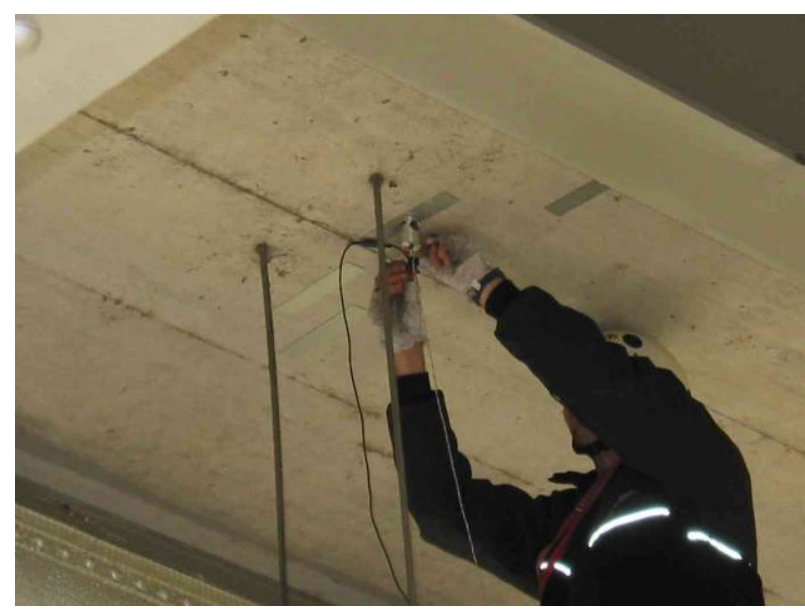

Bild 6 : Anbringen eines Reibungsmessstreifens an der Unterseite der Betonplatte 


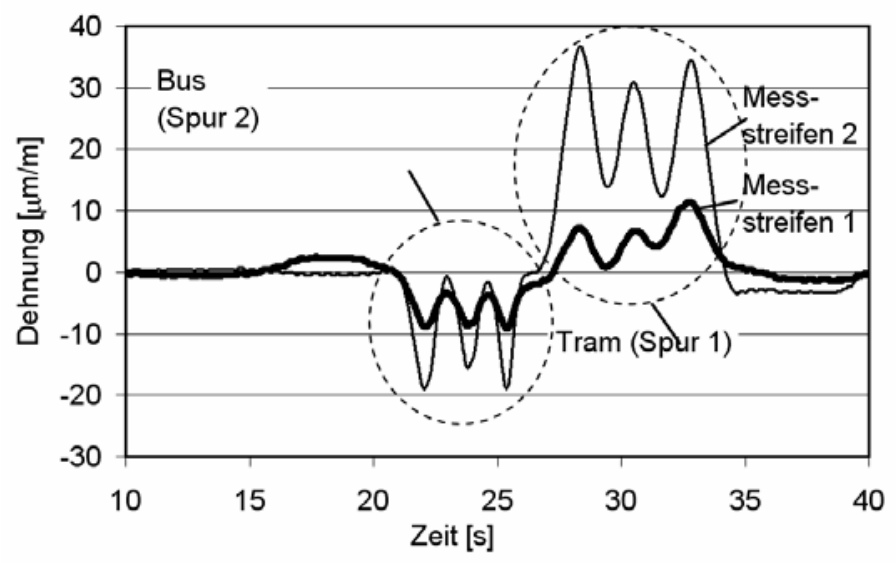

Bild 7 : Messergebnisse von Verformungen (mit 2 Messstreifen) an der Diagonale eines Querträgers während der Überfahrt zweier sich kreuzender Fahrzeuge (Bus und Tram)

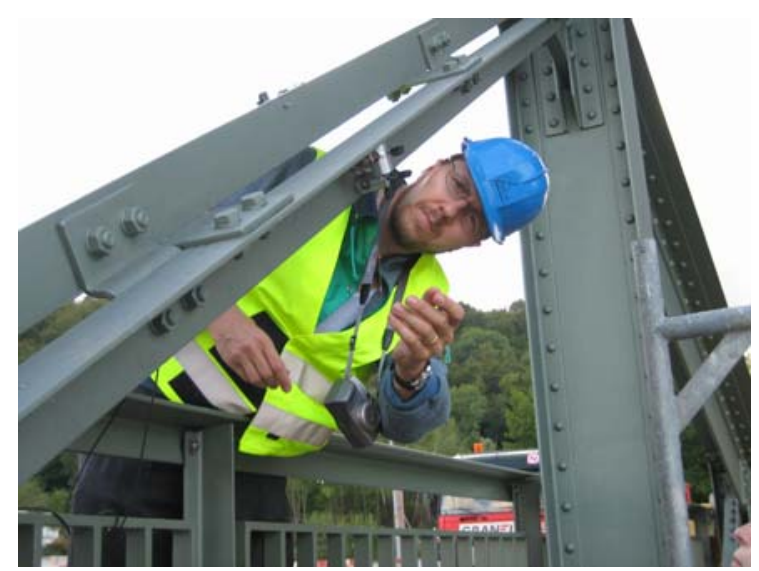

Bild 8 : Anbringen der Reibungsmessstreifen an einer Bogendiagonale

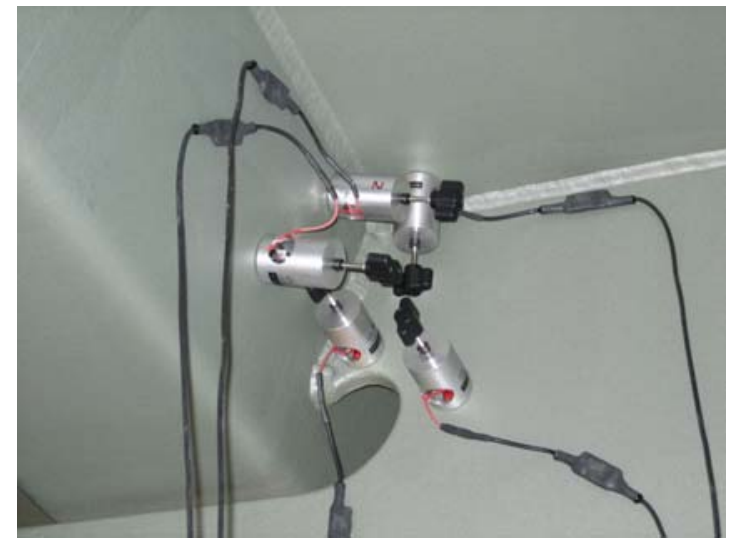

Bild 9 : Reibungsmessstreifen an der orthotropen Platte 